\title{
Prevalência da incontinência urinária em adultos e idosos hospitalizados
}

\author{
PREVALENCE OF URINARY INCONTINENCE IN HOSPITALIZED PATIENTS
}

PREVALENCIADE INCONTINENCIA URINARIA EN PACIENTES HOSPITALIZADOS

\section{Anderson Peterson Machado da Silva ${ }^{1}$, Vera Lúcia Conceição de Gouveia Santos²}

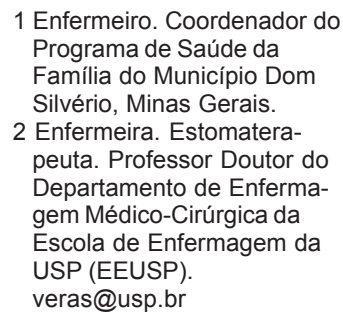

1 Enfermeiro. Coordenador do Programa de Saúde da

Família do Município Dom Silvério, Minas Gerais.

2 Enfermeira. Estomaterapeuta. Professor Doutor do Departamento de Enfermagem Médico-Cirúrgica da Escola de Enfermagem da USP (EEUSP).

veras@usp.br

\begin{abstract}
RESUMO
Este estudo objetivou verificar a prevalência da incontinência urinária em pacientes hospitalizados e identificar as associações estatísticas existentes entre os índices obtidos e algumas variáveis demográficas e clínicas da clientela. Os dados foram colhidos no Hospital Universitário da USP, junto a 77 pacientes internados em três diferentes clínicas. Os resultados indicaram prevalências total de $35 \%$ e parciais de $48 \%$, $37 \%$ e $22 \%$ nas Clínicas Cirúrgica, Obstétrica e Médica, respectivamente. Correlações estatisticamente significativas foram verificadas entre a prevalência e disúria $(\mathrm{r}=0,19$ e $\mathrm{p}=0,046)$, infecções urinárias $(\mathrm{r}=0,24 \mathrm{e}$ $\mathrm{p}=0,019)$, tempo de internação $(\mathrm{r}=-0,32$ e $\mathrm{p}=0,002)$ e sexo masculino $(\mathrm{r}=-0,27$ e $\mathrm{p}=0,008)$.
\end{abstract}

\section{DESCRITORES}

Incontinência urinária (epidemiologia).

Pacientes internados.

\begin{abstract}
The aim of this study is to verify the prevalence of urinary incontinence in hospitalized patients and identify the statistical associations between the figures gathered and some of the patients' demographic and clinical variables. The data were obtained with 77 inpatients of three different units from the University of São Paulo's Hospital Universitário. The results showed a total prevalence of $35 \%$ and partial rates of $48 \%, 37 \%$ and $22 \%$ in the Surgical, Obstetric and Clinical Units respectively. Significant statistical correlation were observed between the prevalence and dysuria $(r=0.19$ and $\mathrm{p}=0.046$ ), urinary infections $(r=0.24$ and $\mathrm{p}=0.019)$, length of inpatient stay $(\mathrm{r}=-0.32$ and $\mathrm{p}=0.002$ ) and gender (males: $\mathrm{r}=-0.27$ and $\mathrm{p}=0.008$ ).
\end{abstract}

\section{KEY WORDS}

Urinary incontinence

(epidemiology).

Inpatient.

\section{RESUMEN}

El estudio objetiva verificar la prevalencia de la incontinencia urinaria en pacientes ingresados e identificar las relaciones estadísticas entre la prevalencia y los datos demográficos y clínicos de los pacientes. Los datos fueron obtenidos de 77 pacientes de tres unidades diferentes del Hospital Universitario de la Universidad de São Paulo (USP). Los resultados apuntaron prevalencia total del $35 \%$ e índices parciales de $48 \%, 37 \%$ y $22 \%$, respectivamente. Correlaciones estadísticas significativas fueron verificadas entre el índice de prevalencia y disuria $(\mathrm{r}=0,19$ and $\mathrm{p}=0,046$ ), infección urinária $(\mathrm{r}=0,24$ y $\mathrm{p}=0,019)$, tiempo de ingreso $(\mathrm{r}=-0,32$ y $\mathrm{p}=0,002) \mathrm{y}$ sexo masculino $(\mathrm{r}=-0,27 \mathrm{y}$ $\mathrm{p}=0,008)$.

\section{DESCRIPTORES}

Incontinencia urinaria (epidemiología).

Ingreso de pacientes. 


\section{INTRODUÇÃO}

No Brasil, o tema da Incontinência Urinária (IU) tem sido pouco explorado, detendo-se principalmente às publicações na área médica, relacionadas aos métodos diagnósticos, terapêuticos e cirúrgicos, permanecendo escassos os estudos sobre os seus dados de prevalência e incidência e restringindo-se a grupos populacionais específicos como diabéticos, idosos, mulheres e outros.

A International Continence Society (ICS) define IU como "uma condição na qual a perda involuntária de urina é um problema social ou higiênico e é objetivamente demonstrável”(1-2).

Vários fatores têm sido relacionados à ocorrência de sintomas da IU, sendo considerados os mais importantes a idade avançada, a gravidez, o parto, a queda dos níveis de estrógeno na menopausa, o tratamento de câncer de próstata, as incapacidades física e mental e algumas doenças prevalentes em idosos como o Acidente Vascular Cerebral e o Mal de Parkinson, além de medicações e cirurgias que são potencialmente capazes de provocar a diminuição do tônus muscular pélvico e/ ou gerar danos nervosos ${ }^{(3-4)}$.

Recentemente, a IU vem sendo classificada a partir dos sintomas específicos referidos pelos pacientes, como incontinência total, por estresse, de transbordamento, funcional e mista ${ }^{(2,5-7)}$.

No que diz respeito às formas de tratamento, podem ser do tipo cirúrgico e não cirúrgico. $\mathrm{O}$ tratamento cirúrgico envolve uma série de técnicas, destacando-se o "Sling" de parede vaginal, a Colposuspensão de Burch e a suspensão endoscópica de colo vesical ${ }^{(5)}$. Porém, o tratamento não cirúrgico tem sido a terapia de primeira escolha e baseia-se na utilização de fármacos como os agentes anticolinérgicos, os relaxantes musculotrópicos, os abridores de canais de potássio, os inibidores de prostaglandinas, os agonistas beta e alfa adrenérgicos, os antagonistas betaadrenérgicos, o estrógeno e o hormônio antidiurético. Ele inclui também a fisioterapia do assoalho pélvico e a utilização de recursos mecânicos. Cabe ressaltar que estas técnicas podem ser empregadas isoladamente ou de forma combinada ${ }^{(8-9)}$.

Quanto aos índices que apontam a prevalência e incidência da IU, estes dependem das características da população estudada como a idade, o sexo, as condições físicas e mentais, além da definição de incontinência adotada e das condições de saúde geral da população alvo ${ }^{(7)}$.
Em 1994, segundo a National Kidney and Urologic Disease Advisory Board, a IU afetava 13 milhões de americanos, com elevadas freqüências entre os idosos. Cerca de $10 \%$ a $35 \%$ dos adultos eram considerados incontinentes, sendo que grande parte destes recebia assistência domiciliária. Diante disto, a IU tem representado, anualmente para os EUA, gastos da ordem de $\$ 11,2$ bilhões, sendo 5,2 bilhões relacionados às "nursing homes" ${ }^{\prime \prime}$.

Ao gerar implicações negativas nos âmbitos emocional, social e econômico tanto para o indivíduo incontinente, como para seus amigos, familiares e cuidadores, a IU representa um desafio para profissionais da saúde, na busca de formas alternativas de abordagem e tratamento do problema, o que tem sido constantemente subestimado, principalmente em nosso meio.

Reconhecendo-se que o estabelecimento de um diagnóstico dos índices de prevalência e incidência de IU em diferentes grupos populacionais deve anteceder a proposição de intervenções preventivas e terapêuticas, além da carência de trabalhos nacionais relacionados a tal problemática, desenvolveu-se este estudo com os seguintes objetivos: identificar a prevalência da IU em pacientes adultos e idosos hospitalizados e verificar as associações estatísticas existentes entre os índices encontrados e as variáveis sócio-demográficas, clínicas e ligadas às perdas urinárias da população estudada.

\section{CASUÍSTICA E MÉTODO}

Este estudo, do tipo descritivo, exploratório e transversal, com abordagem quantitativa, foi desenvolvido no Hospital Universitário da Universidade de São Paulo (HUUSP), localizado no município de São Paulo.

A população foi constituída de todos os pacientes adultos que se encontravam internados nas Clínicas Médica (CM), Cirúrgica (CC) e Centro Obstétrico (CO), durante o período de coleta de dados, totalizando 77 indivíduos que se enquadravam nos critérios de seleção: ter idade igual ou superior a 18 anos, ter condições físicas e mentais para responder à entrevista e consentir em participar do estudo.

A coleta de dados foi realizada em três dias consecutivos, de terça a quinta- feira, precedida pela aprovação do projeto de pesquisa pelo Comitê de Ética do HU-USP.
Prevalência da incontinência urinária em adultos e idosos hospitalizados 
Anderson P. M. da Silva Vera Lúcia C. G. Santos
Antes de proceder-se à coleta de dados em cada unidade, solicitava-se a relação dos pacientes internados e, somente após a definição dos pacientes elegíveis, passava-se aos esclarecimentos sobre os objetivos da pesquisa, solicitando-se a assinatura do termo de consentimento e posterior entrevista. Esta foi direcionada por um instrumento de coleta de dados que abordava os dados sócio-demográficos e clínicos gerais para todos os pacientes, além dos específicos relacionados às perdas urinárias - PU (clínicas, sentimentos e comprometimento nas atividades de vida diária e nas relações afetivas e sociais), para os pacientes que as informavam.

A variável demográfica ocupação foi classificada em 5 níveis que engloba desde ocupação não definida (nível zero) ao profissional liberal e cargos de alto nível administrativo (nível cinco).

Os resultados foram submetidos às análises descritiva e inferencial. A análise descritiva foi realizada para as variáveis sócio-demográficas, clínicas e ligadas às perdas urinárias (PU), ado- tando-se distribuições de freqüência, média, mediana, desvio padrão, mínimo e máximo para a produção de estimativas pontuais, e Intervalos de Confiança de $95 \%$ para estimativas intervalares de algumas características populacionais de interesse. A análise inferencial foi constituída das análises de correlação, associações e comparação entre variáveis de interesse, através dos seguintes procedimentos estatísticos: Intervalos de Confiança de $95 \%$, usados para estimar o valor médio real das variáveis quantitativas e porcentagens reais de alguns eventos de interesse do estudo; Coeficiente de Correlação Ordinal de Sperman e o Coeficiente de Correlação Linear de Pearson para as análises de correlação, Teste de Mann-Whitney, na comparação de duas populações independentes. $P$-value inferior a 0.05 foi considerado para significância estatística.

\section{RESULTADOS}

A Figura 1 apresenta os índices de prevalência da IU, obtidos junto ao total de pacientes entrevistados e por unidade investigada.

\section{$\square$ Clínica Cirúrgica $\square$ Clínica Médica $\square$ Centro Obstétrico $\square$ Total Geral}

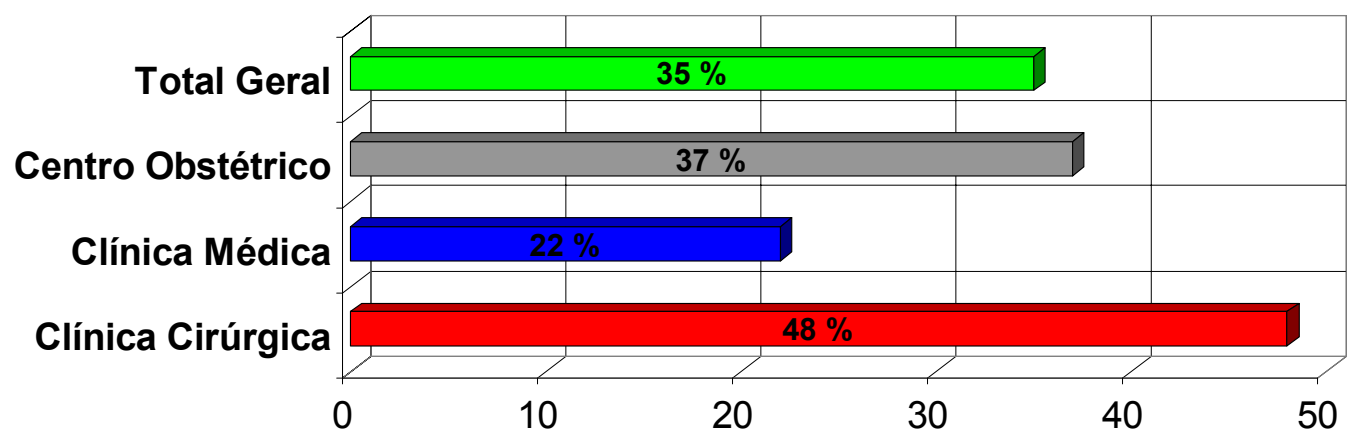

Figura 1 - Prevalência de IU segundo o total de pacientes e a unidade de internação. (São Paulo, 2001)

De um total de 77 pacientes hospitalizados que atendiam aos requisitos para responder à entrevista, 27 apresentavam perdas urinárias, caracterizando uma prevalência total para a IU de $35 \%$, mostrada na Figura 1. Quanto às unidades, os índices variaram de $22 \%$ na $\mathrm{CM}, 37 \%$ no $\mathrm{CO}$ e $48 \%$ na $\mathrm{CC}$, sem diferenças estatisticamente significativas entre eles, de acordo com o Teste Qui- Quadrado de associação.
A seguir, são apresentados os resultados para as variáveis demográficas e clínicas para os grupos com e sem IU, em valores absolutos e percentuais, representados por tabelas e figuras. As características das perdas urinárias são descritas apenas para as pessoas do grupo com IU.
Rev Esc Enferm USP $2005 ; 39(1): 36-45$. 
CARACTERÍSTICA

\begin{tabular}{|c|c|c|c|c|c|c|}
\hline \multirow{3}{*}{ Idade } & \multirow{2}{*}{\multicolumn{2}{|c|}{ S/IU }} & \multirow{2}{*}{\multicolumn{2}{|c|}{$\mathrm{C} / \boldsymbol{I U}$}} & \multirow{2}{*}{\multicolumn{2}{|c|}{ TOTAL }} \\
\hline & & & & & & \\
\hline & $\mathbf{n}$ & $\%$ & $\mathbf{n}$ & $\%$ & $\mathbf{n}$ & $\%$ \\
\hline$<45$ & 26 & 52.0 & 16 & 59.3 & 42 & 54.5 \\
\hline 45 a 60 & 11 & 22.0 & 7 & 25.9 & 18 & 23.4 \\
\hline$>60$ & 13 & 26.0 & 4 & 14.8 & 17 & 22.1 \\
\hline Média (DP) & \multicolumn{2}{|c|}{$44.2(18.7)$} & \multicolumn{2}{|c|}{$40.7(19.1)$} & \multicolumn{2}{|c|}{$43.0(19.0)$} \\
\hline Variaçāo & \multicolumn{2}{|c|}{18 a 83} & \multicolumn{2}{|c|}{18 a 76} & \multicolumn{2}{|c|}{18 a 83} \\
\hline Mediana & \multicolumn{2}{|c|}{43.50} & \multicolumn{2}{|c|}{34} & \multicolumn{2}{|c|}{40} \\
\hline IC $95 \%$ para a Média & \multicolumn{2}{|c|}{$44.2 \pm 5.4$} & \multicolumn{2}{|c|}{$40.7 \pm 7.6$} & \multicolumn{2}{|c|}{43.0} \\
\hline \multicolumn{7}{|l|}{ Sexo } \\
\hline Feminino & 29 & 58.0 & 22 & 81.5 & 51 & 66.2 \\
\hline Masculino & 21 & 42.0 & 5 & 18.5 & 26 & 33.8 \\
\hline IC $95 \%$ p/a \% Mase & \multicolumn{2}{|c|}{$42.0 \pm 14.2$} & \multicolumn{2}{|c|}{$18.5 \pm 15.7$} & \multicolumn{2}{|c|}{$33.8 \pm 10.8$} \\
\hline \multicolumn{7}{|l|}{ Etnia } \\
\hline Branca & 38 & 76.0 & 19 & 70.4 & 57 & 74.0 \\
\hline Negra & 1 & 2.0 & - & - & 1 & 1,3 \\
\hline Amarela & 11 & 22.0 & 8 & 29.6 & 19 & 24.7 \\
\hline \multicolumn{7}{|l|}{ Escolaridade } \\
\hline Analfabeto & 7 & 14.0 & 4 & 14.8 & 11 & 14.3 \\
\hline Ensino Fundamental & 23 & 46.0 & 16 & 59.3 & 39 & 50.6 \\
\hline Ensino Médio & 12 & 24.0 & 6 & 22.2 & 18 & 23.4 \\
\hline Ensino Superior & 8 & 16.0 & 1 & 3.7 & 9 & 11.7 \\
\hline Ocupação Anterior (Fonse & & & & & & \\
\hline Nível 0 & 29 & 58.0 & 21 & 77.8 & 50 & 64.9 \\
\hline Nível 1 & 8 & 16.0 & 3 & 11.1 & 11 & 14.3 \\
\hline Nível 2 & 4 & 8.0 & 1 & 3.7 & 5 & 6.5 \\
\hline Nível 3 & 1 & 2.0 & 2 & 7.4 & 3 & 3.9 \\
\hline Nível 4 & 4 & 8.0 & - & - & 4 & 5.2 \\
\hline Nível 5 & 4 & 8.0 & - & - & 4 & 5.2 \\
\hline Estado Civil & & & & & & \\
\hline C/companheiro & 33 & 66.0 & 15 & 55.6 & 48 & 62.3 \\
\hline S/companheiro & 17 & 34.0 & 12 & 44.4 & 29 & 37.7 \\
\hline Renda (salários mínimos & & & & & & \\
\hline$\leq 3$ & 15 & 30.0 & 16 & 59.3 & 31 & 40.3 \\
\hline 4 a 5 & 21 & 42.0 & 9 & 33.3 & 30 & 39.0 \\
\hline 6 a 10 & 11 & 22.0 & 2 & 7.4 & 13 & 16.9 \\
\hline$>10$ & 3 & 6.0 & - & - & 3 & 3.9 \\
\hline Número de Dependente: & & & & & & \\
\hline$\leq 3$ & 29 & 58.0 & 11 & 40.7 & 40 & 52.0 \\
\hline 4 a 6 & 17 & 34.0 & 12 & 44.4 & 29 & 37.7 \\
\hline$>6$ & 4 & 8.0 & 4 & 14.8 & 8 & 10.4 \\
\hline Média (DP) & & & & & & \\
\hline Variação & & & & & & \\
\hline Mediana & & & & & & \\
\hline IC $95 \%$ para a Média & 3.8 & & & & & \\
\hline
\end{tabular}

Prevalência da incontinência urinária em adultos e idosos hospitalizados
Os dados da Tabela 1 mostram que, dos 77 pacientes que participaram do estudo, $42(54,5 \%)$ possuíam menos de 45 anos de idade, caracterizando a média de 43 anos ( $\mathrm{DP}=19,0) ; 51(66,2 \%)$ eram do sexo feminino e $57(74,0 \%)$ pertenciam à étnica branca. Além destas características, a maioria possuía baixo nível de escolaridade (39 ou 50,6\% no nível fundamental), ocupações do nível zero (50 ou $64,9 \%$ ) e com companheiros ( 48 ou $62,3 \%$ ). Características semelhantes foram observadas no interi- or dos grupos sem e com IU, exceto para o sexo no qual, embora com predomínio de mulheres em ambos grupos, elas constituíram a grande maioria $(81,5 \%)$ entre os pacientes do grupo com IU.

Apesar da renda salarial média variar conforme o grupo, verifica-se que se concentrou na faixa até 5 salários mínimos, com números médios de dependentes de 3,8 $(\mathrm{DP}=1,7)$ para os pacientes sem $\mathrm{IU}$, e de $4,5(\mathrm{DP}=1,8)$ para aqueles incontinentes. 
Anderson P. M. da Silva Vera Lúcia C. G. Santos

Tabela 2 - Características clínicas da população estudada. (São Paulo, 2001)

\begin{tabular}{|c|c|c|c|c|c|c|}
\hline \multirow{3}{*}{$\begin{array}{c}\text { VARIÁVEIS } \\
\text { Motivo da Internação }\end{array}$} & \multicolumn{6}{|c|}{ DISTRIBUIÇÃO } \\
\hline & \multicolumn{2}{|c|}{$\mathbf{S} / \mathbf{T U}$} & \multicolumn{2}{|c|}{$\mathrm{C} / \mathbf{T U}$} & \multicolumn{2}{|c|}{ Global } \\
\hline & $\mathrm{n}$ & $\%$ & $\mathrm{n}$ & $\%$ & $\mathrm{n}$ & $\%$ \\
\hline Digestório & 8 & 16.0 & 2 & 7.4 & 10 & 13.0 \\
\hline Uroginecológico & 2 & 4.0 & 4 & 14.8 & 6 & 7.8 \\
\hline Resp. Cardiovascular & 11 & 22.0 & 4 & 14.8 & 15 & 19.5 \\
\hline Metabólico & 3 & 6.0 & - & - & 3 & 3.9 \\
\hline Obstétrico & 16 & 32.0 & 8 & 29.6 & 24 & 31.2 \\
\hline Outros & 10 & 20.0 & 9 & 33.3 & 19 & 24.6 \\
\hline \multicolumn{7}{|l|}{ T. Internação } \\
\hline$\leq 15$ & 40 & 80.0 & 25 & 92.6 & 65 & 84.4 \\
\hline $1 \overrightarrow{6}$ a 30 & 2 & 4.0 & 1 & 3.7 & 3 & 3.9 \\
\hline$\geq 31$ & 8 & 16.0 & 1 & 3.7 & 9 & 11.7 \\
\hline Média (DP) & 13.0 & & $7(10.8$ & & $11.0(15$ & \\
\hline Variação & 1 a 68 & & 2 a 58 & & 1 a 6 & \\
\hline Mediana & 6 & & 4 & & 5 & \\
\hline IC $95 \%$ para a Média & 13.0 & & & & 11.0 & \\
\hline \multicolumn{7}{|l|}{ Cirurgia Uroginecológica } \\
\hline Não & 44 & 91.7 & 20 & 74.1 & 64 & 85.3 \\
\hline Sim & 4 & 8.3 & 7 & 25.9 & 11 & 14.7 \\
\hline IC $95 \%$ para \%Sim & \multicolumn{2}{|c|}{$8.3 \pm 8.1$} & \multicolumn{2}{|c|}{$25.9 \pm 17.7$} & \multicolumn{2}{|c|}{$14.7 \pm 8.2$} \\
\hline \multicolumn{7}{|c|}{ Número de Partos Vaginais } \\
\hline$<4$ & 20 & 40.0 & 12 & 44.4 & 32 & 41.6 \\
\hline 4 a 6 & 1 & 2.0 & 6 & 22.2 & 7 & 9.1 \\
\hline$>6$ & 29 & 58.0 & 9 & 33.4 & 38 & 49.3 \\
\hline Média (DP) & \multicolumn{2}{|c|}{$1.8(3.2)$} & \multicolumn{2}{|c|}{$2.6(3,0)$} & \multicolumn{2}{|c|}{$2.2(3.1)$} \\
\hline Variação & \multicolumn{2}{|c|}{0 a 11} & \multicolumn{2}{|c|}{0 a 12} & \multicolumn{2}{|c|}{0 a 12} \\
\hline Mediana & \multicolumn{2}{|c|}{0} & \multicolumn{2}{|c|}{3} & \multicolumn{2}{|c|}{0} \\
\hline IC $95 \%$ para a Média & \multicolumn{2}{|c|}{$1.8 \pm 1.4$} & \multicolumn{2}{|c|}{$2.6 \pm 1.4$} & \multicolumn{2}{|c|}{$2.2 \pm 0.9$} \\
\hline Perda Urinária & & & & & & \\
\hline Não & 40 & 80.0 & 5 & 18.5 & 45 & 58.4 \\
\hline Sim Recentemente & 6 & 12.0 & 22 & 81.5 & 28 & 36.4 \\
\hline Sim no Passado & 4 & 8.0 & - & - & 4 & 5.2 \\
\hline Disúria & & & & & & \\
\hline Não & 33 & 66.0 & 16 & 59.3 & 49 & 63.6 \\
\hline Sim Recentemente & 13 & 26.0 & 10 & 37.0 & 23 & 29.9 \\
\hline Sim no Passado & 4 & 8.0 & 1 & 3.7 & 5 & 6.5 \\
\hline Infecção & & & & & & \\
\hline Não & 39 & 78.0 & 16 & 59.3 & 55 & 71.4 \\
\hline Sim & 9 & 18.0 & 10 & 37.0 & 19 & 24.7 \\
\hline Não Sabe Informar & 2 & 4.0 & 1 & 3.7 & 3 & 3.9 \\
\hline PU Atualmente & & & & & & \\
\hline Não & 50 & 100.0 & - & - & 50 & 64.9 \\
\hline Sim & - & - & 27 & 100.00 & 27 & 35.1 \\
\hline IC $95 \%$ para a \% Sim & 0.0 & & 100 & 0.0 & 35 & \\
\hline
\end{tabular}

Verifica-se, através dos resultados da Tabela 2 , que as causas obstétricas corresponderam a cerca de 1/3 dos motivos das internações, tanto para o total de pacientes como para ambos grupos - sem e com IU - $(31,2 \% ; 32,0 \%$ e $29,6 \%$, respectivamente). $\mathrm{O}$ tempo de internação foi igual ou inferior a 15 dias para ambos grupos, porém o tempo médio foi inferior para o grupo de pacientes com IU ( 7 dias e $\mathrm{DP}=10,8$ ) comparativamente àqueles sem IU (13,0 e DP = 17,3), com diferença significativa entre eles.
Quanto a alguns fatores de risco ou associados às perdas urinárias, constatou-se que a maioria dos pacientes (64 ou 85,3\%) não referiu cirurgias uroginecológicas prévias, o que se repetiu no interior dos grupos, apesar de um índice menor para os pacientes incontinentes $(74,1 \%)$ comparativamente aos continentes $(91,7 \%)$. O número médio de partos vaginais e a mediana são ligeiramente superiores para os pacientes com IU ( 2,6 e DP $=3,0$ e 3,0 respectivamente para média, DP e mediana). Predominaram também a
Rev Esc Enferm USP $2005 ; 39(1): 36-45$. 
ausência de queixas para a disúria e infecções urinárias tanto para o total de pacientes $(63,6 \% \mathrm{e}$ $71,4 \%$, respectivamente) como no interior de ambos grupos $(66,0 \%$ e $78,0 \%$ para os pacientes sem IU; e 59,3\% e 59,3\% para os pacientes com IU).
As características e percepções acerca das perdas urinárias (PU) de 27 pacientes que as referiram atualmente, encontram-se descritas na Tabela 3.
Prevalência da incontinência urinária em adultos e idosos hospitalizados

Tabela 3 - Características das perdas urinárias. (São Paulo, 2001)

\begin{tabular}{|c|c|c|}
\hline VARIÁVEL & \multicolumn{2}{|c|}{ DISTRIBUIÇÃO } \\
\hline Tempo (anos) & $\mathbf{n}$ & $\%$ \\
\hline$<1$ & 12 & 44.5 \\
\hline 1 a 3 & 9 & 33.3 \\
\hline 4 a 6 & 2 & 7.4 \\
\hline$\geq 6$ & 4 & 14.8 \\
\hline \multicolumn{3}{|l|}{ Cons. Chegar ao Banheiro } \\
\hline Sempre & 17 & 63.0 \\
\hline Na Maioria das Vezes & 2 & 7.4 \\
\hline As Vezes & 4 & 14.8 \\
\hline Nunca & 4 & 14.8 \\
\hline \multicolumn{3}{|l|}{ Acorda para urinar } \\
\hline Não & 2 & 7.4 \\
\hline Sim e vai ao Banheiro & 23 & 85.2 \\
\hline Sim e não vai ao Banheiro & 2 & 7.4 \\
\hline \multicolumn{3}{|l|}{ Condições } \\
\hline Pequenos Esforços & 7 & 25.9 \\
\hline Médios Esforços & 18 & 66.7 \\
\hline Grandes Esforços & 2 & 7.4 \\
\hline \multicolumn{3}{|l|}{ Durante Sexo } \\
\hline Não & 24 & 88.9 \\
\hline Sim & 3 & 11.1 \\
\hline IC95\% para \%Sim & $11.1 \pm 12.7$ & \\
\hline \multicolumn{3}{|l|}{ Freqüência } \\
\hline Esporádica & 11 & 40.7 \\
\hline Semanal & 7 & 25.3 \\
\hline \multicolumn{3}{|l|}{ Recursos } \\
\hline Absorvente & 2 & 28.6 \\
\hline Fraldas & 2 & 28.6 \\
\hline Outros & 3 & 42.8 \\
\hline \multicolumn{3}{|l|}{ Troca de Recursos } \\
\hline Uma Vez ao Dia & 5 & 71.4 \\
\hline Duas Vezes ao Dia & 1 & 14.3 \\
\hline Três ou Mais Vezes ao Dia & 1 & 14.3 \\
\hline \multicolumn{3}{|l|}{ Mudancas de Hábito } \\
\hline Não & 22 & 81.5 \\
\hline $\operatorname{Sim}$ & 5 & 18.5 \\
\hline IC 95\% para \% Sim & $18.5 \pm 15.7$ & \\
\hline \multicolumn{3}{|l|}{ Mudanças de Ocupação } \\
\hline Não & 26 & 96.3 \\
\hline Sim & 1 & 3.7 \\
\hline IC95\% para \% Sim & $3.7 \pm 7.6$ & \\
\hline
\end{tabular}

A Tabela 3 mostra que 21 pacientes $(77,8 \%)$ apresentavam perdas urinárias há até 3 anos, 12 dos quais (44,5\%) há menos de 1 ano. Apesar das perdas, a maioria dos pacientes referiu que conseguia chegar sempre ao banheiro ( 17 ou $63,0 \%$ ), não apresentava perdas durante as relações sexuais (24 
Anderson P. M. da Silva Vera Lúcia C. G. Santos ou $88,9 \%$ ) e não apresentou mudanças de hábitos de vida diária ( 22 ou $81,5 \%$ ) ou de ocupação ( 26 ou $96,3 \%$ ) como conseqüência das PU. Além dessas características, as perdas eram esporádicas para 11 ou $40,7 \%$ deles; apenas 7 (25,9\%) utilizavam recursos de contenção, trocados apenas $1 \mathrm{vez} /$ dia para 5 deles. As PU foram consideradas como ocorrências aos médios esforços (18 ou $66,7 \%)$ e 23 pacientes $(85,2 \%)$ acordavam à noite e iam ao toalete para urinar.

À pergunta acerca dos sentimentos relacionados à IU, somente 8 pacientes apontaram 22 respostas, das quais a mais mencionada foi a vergonha (8). Ansiedade, medo e desconforto também foram mencionados, com 4 respostas cada um. Vinte e três pacientes informaram ausência de comprometimento nas relações sociais e afetivas devido às PU. Verificaram-se, no entanto, 6 respostas indicativas de influência negativa nas relações com amigos e familiares, na sexualidade e no campo profissional. Quanto à percepção sobre a gravidade da IU, 19 pacientes $(70,4 \%)$ a percebem como sem gravidade, enquanto os demais ( 8 ou $29,6 \%$ ) consideram-na como grave ou muito grave.

$\mathrm{Na}$ análise inferencial, constataram-se algumas correlações estatisticamente significativas, de fraca a moderada intensidade, entre o índice de prevalência e o tempo de internação $(\mathrm{r}=-0,32$ e $\mathrm{P}=0,002)$, sexo masculino $(\mathrm{r}=-0,27 \mathrm{e} \mathrm{P}=0,008)$, dificuldade para urinar $(\mathrm{r}=0,19$ e $\mathrm{P}=0,046)$ e a presença de infecção $(\mathrm{r}=0,24 \mathrm{e} \mathrm{P}=0,019)$. Desse modo, os achados sugerem que maiores índices de prevalência correlacionam-se com os menores tempos de internação, com o sexo feminino e com as presenças de disúria e infecção urinárias prévias.

\section{DISCUSSÃO}

Poucos trabalhos científicos têm se dedicado ao estudo da incontinência urinária em pacientes hospitalizados, o que dificulta a comparação dos resultados encontrados neste estudo com aqueles divulgados na literatura, geralmente relacionados a grupos populacionais específicos como idosos, mulheres e diabéticos. É possível constatar no entanto que, mesmo diante das diferentes abordagens do tema, existe um consenso de que a IU é um problema que deve merecer mais atenção por parte dos profissionais da saúde, visto os altos índices de prevalência encontrados.

Os resultados relacionados à prevalência da IU em adultos e idosos hospitalizados no HU-USP, a partir de entrevistas com 77 pacientes, mostraram índices de $48 \%$ na CC, $37 \%$ no CO, $22 \%$ na $\mathrm{CM}$ e prevalência global de $35 \%$, sem diferenças estatisticamente significativas entre eles.

Em recente estudo sobre a qualidade do cuidado específico relacionado à IU, obteve-se índices diferenciados de prevalência junto a um total de 1125 londrinos, conforme o tipo de unidade de longa permanência investigado ${ }^{(10)}$. Assim, encontraram 34\% de pessoas com IU em 17 "residential homes", 70\% em 14 instituições asilares e $71 \%$ em 5 unidades de longa permanência. Em estudo similar japonês com 9798 pacientes institucionalizados, com idades, igual e superior a 60 anos, obteve-se prevalências de $23,3 \%$ e $23,8 \%$, respectivamente para homens e mulheres hospitalizados, valores muito inferiores àqueles apresentados por pessoas no grupo de asilados, ou seja, $64,2 \%$ e $67,9 \%$ para homens e mulheres, respectivamente ${ }^{(11)}$. Diferentemente desses autores, em outra pesquisa realizada no Japão, apontou índices bem inferiores de apenas $15 \%$ de IU em 1023 idosos institucionalizados, sendo 8\% para pacientes do sexo masculino e $19 \%$, para o sexo feminino ${ }^{(12)}$. Ainda junto a pacientes geriátricos, encontraram $54,5 \%$ como prevalência de IU em 839 pessoas em "residential homes" e instituições asilares no norte da Itália, com aumento significativo conforme a idade ${ }^{(13)}$. Novamente as mulheres apresentaram índice bastante elevado $(59,8 \%)$ comparativamente aos homens $(39,2 \%)$. Em outro estudo multicêntrico, a comparação dos resultados encontrados na República Tcheca ${ }^{(14)}$ com aqueles presentes no banco de dados INTERRAI provenientes de outros sete países (Dinamarca, França, Irlanda, Itália, Japão, Suécia e EUA), apontam que a República Tcheca está entre aqueles com os mais elevados índices de prevalência tanto para IU como para incontinência anal, havendo uma variação de $42,9 \%$ no Japão a $65,2 \%$ na França.

Para estudos na população geral, destacamse o Inquérito Nacional de Saúde, realizado em Portugal, no ano de 1995, quando foi detectada prevalência global de $4,3 \%{ }^{(15)}$. Pesquisadores espanhóis, em 2000, obtiveram valores bem mais elevados por tratarem-se de 827 idosos na zona rural da Espanha $(36 \%)^{(16)}$. O mesmo ocorreu em estudo longitudinal com 2087 idosos no sul da Austrália ${ }^{(17)}$, que encontraram IU de urgência em $36,6 \%$ a $41,6 \%$ das pessoas, e IU de esforço em $23,4 \%$ a $28,8 \%$ dos indivíduos, ao longo dos dois anos de seguimento.

Ao serem detalhadas as características sócio-demográficas da clientela com IU, destacam- 
se a idade e o sexo como variáveis que podem estar relacionadas às PU. Em uma amostra, cuja idade variou de 18 a 83 anos, as médias etárias foram similares entre os grupos de pacientes com e sem IU, apesar de mediana inferior para os pacientes incontinentes quando comparada àquela apresentada pelos pacientes sem IU. Nesse sentido, a literatura é rica ao relacionar a idade avançada à presença de alguns tipos de IU ${ }^{(9,18-22)}$, tanto em função do processo normal de envelhecimento como da co-existência de doenças sistêmicas que favoreceriam a ocorrência das perdas urinárias, o que não foi verificado neste estudo.

Já, para o sexo, o amplo predomínio das mulheres no grupo dos pacientes incontinentes $(81,5 \%)$ comparativamente ao grupo sem IU, além da presença de correlação estatisticamente significativa com o índice de prevalência, confirmam, mais uma vez, os achados de inúmeros outros autores quanto às maiores freqüências de perdas urinárias entre as mulheres ${ }^{(9,19-23)}$. A investigação da presença de IU em 2000 idosos de uma cidade inglesa obtiveram prevalências sempre superiores nas mulheres de todas as idades, comparativamente aos homens (42\% e $34 \%$, respectivamente para mulheres e homens acima de 80 anos) ${ }^{(24)}$. No estudo epidemiológico realizado em Portugal ${ }^{(15)}$, a IU crônica foi referida por $3,1 \%$ de todas as pessoas investigadas, com índices de 1,8\% para os homens e $4,2 \%$ para as mulheres. Pesquisadores brasileiros, investigando os conhecimentos e atitudes sobre a IU junto a 400 pessoas da cidade de São Paulo, encontraram IU em 142 delas, das quais 77,46\% eram mulheres ${ }^{(23)}$.

Clinicamente, alguns achados de nosso estudo devem ser comentados, mesmo que não tenham implicado em diferenças estatisticamente significativas entre os grupos. Desse modo, embora a maioria dos entrevistados não tenha referido cirurgias uroginecológicas anteriores, disúria ou infecções urinárias, verificaram-se maiores percentuais dessas condições para os pacientes com IU. Além disso, correlações positivas estatisticamente significativas foram detectadas entre os índices de prevalência e disúria, indicando tais condições como fatores que podem estar associados à IU.

A infecção do trato urinário é considerada como um dos fatores concorrentes ${ }^{(22)}$ ou mesmo causais da $\mathrm{IU}^{(19)}$ à medida que piora o comportamento da bexiga já instável, ao aumentar o número e a freqüência de suas contrações involuntárias ${ }^{(22)}$. Disúria foi relatada em amplo estudo realizado junto a todas as mulheres com idade maior ou igual a 19 anos, vinculadas a programas de saúde da família em Bristol $^{(25)}$, principalmente entre as mais jovens.
Quanto ao número de partos vaginais, fator ainda controverso na literatura como fator relacionado ao surgimento da IU, particularmente a incontinência urinária de esforço - IUE ${ }^{(26-28)}$, também obtivemos média e mediana superiores entre as mulheres com IU. Em estudos sobre a qualidade de vida e auto-estima de mulheres com IUE ${ }^{(29-30)}$, encontraram números médios de partos vaginais mais elevados (3,38 e 3,57, respectivamente). Apesar desses achados e embora a IU seja considerada uma situação que afeta principalmente as mulheres multíparas, é também um problema comum entre mulheres nulíparas jovens, durante a realização de exercícios físicos ${ }^{(1,5,7)}$.

Para as características da IU, alguns autores sugerem que um índice de gravidade deveria considerar a combinação de dados como a freqüência e quantidade das perdas urinárias ${ }^{(31)}$. Ao analisarmos os resultados predominantemente obtidos para as características aqui investigadas como conseguir chegar sempre ao banheiro (63,0\%), ausência de perdas durante as relações sexuais (88,9\%), freqüência de perdas (esporádicas para $40,7 \%)$, uso $(25,9 \%)$ e troca de recursos de contenção (1 vez ao dia para 71,4\% dos que utilizam) e ausência de mudanças de hábitos de vida diária $(81,5 \%)$ e de ocupação $(96,3 \%)$ em conseqüência à IU, poderiam ser indicativos de uma IU leve. Somente dois aspectos apontam para uma condição mais grave de IU na clientela deste estudo como as perdas aos médios esforços e o fato de acordar à noite e ter que urinar. Pesquisadores espanhóis encontraram $45 \%, 33 \%$ e $22 \%$ respectivamente de perdas urinárias consideradas leve, moderada e grave para os idosos que referiram $\mathrm{IU}^{(16)}$. Estes valores correspondiam respectivamente a 16\%, 11\% e $9 \%$ da população rural na Espanha. Similarmente aos nossos achados, os mesmos autores obtiveram predomínio de perdas aos médios esforços para $66,67 \%$ dos entrevistados.

Nictúria foi o décimo sintoma mais freqüen-temente mencionado pelas mulheres entrevistadas e 29\% dos 1277 que reportaram perdas urinárias utilizavam recursos de contenção como absorventes e outros produtos, incluindo a troca de roupas íntimas ou externas nos casos mais graves ${ }^{(25)}$. Detectou-se o predomínio de perdas urinárias aos médios esforços (80\%), sensação de umidade (50\%) e utilização de recursos de contenção $(80 \%)$ com trocas diárias (46\%) para as 50 mulheres que compuseram a amostra do estudo ${ }^{(29)}$. Outros autores também encontraram perdas diárias $(60,78 \%)$, aos esforços moderados (56\%), sensação de estar molhada $(43,13 \%)$, utilizando recursos de contenção $(62,74 \%)$, trocados 2 ou mais vezes ao dia $(50,98 \%)$
Prevalência da incontinência urinária em adultos e idosos hospitalizados 
Anderson P. M. da Silva Vera Lúcia C. G. Santos e com mudanças nos hábitos de vida diária $(70,58 \%)$, indicando maior gravidade para as 51 mulheres com IUE, por elas investigadas ${ }^{(30)}$.

Embora sem constituir foco central deste estudo, ao serem questionados acerca de algumas percepções frente à IU, além da ausência de modificações nos hábitos de vida diária e de ocupação provocadas pela IU, esta foi representada como ocorrência sem gravidade para 19 pessoas e que, sobretudo, não interferia nas relações sociais com amigos e familiares, profissionais e sexuais. Apesar disso, alguns sentimentos negativos foram apontados, sendo a vergonha o mais freqüente, seguido de ansiedade, medo e desconforto. Sentimentos negativos que vão do desconforto à depressão, em diferentes níveis de intensidade, e expressos por pessoas com IU de qualquer natureza, são citados por inúmeros autores $^{(6,9,15,22)}$, que podem também em menor ou maior grau comprometer a auto-estima ${ }^{(30)}$ e a qualidade de vida da clientela ${ }^{(29)}$. Apesar dessas possíveis conseqüências, vários outros estudos têm demonstrado como programas multidisciplinares de assistência, envolvendo protocolos e estratégias reabilitatórias, principalmente junto a grupos específicos como idosos instituciona$\operatorname{lizados}^{(32)}$, populações de risco ${ }^{(20)}$ e cuidadores formais ${ }^{(33)}$, podem resultar em controle adequado das perdas urinárias.

\section{CONCLUSÕES}

Este estudo sobre a prevalência de IU em pacientes hospitalizados em três diferentes unidades de internação do HUUSP, em São Paulo, permitiu detectar o problema em 27 dentre 77 pessoas em condições de responder à entrevista, acarretando prevalência global de 35\%. Importantes correlações estatisticamente significativas foram

\section{REFERÊNCIAS}

(1) Doughty DB, Waldrop J. Introductory concepts. In: Doughty DB. Urinary \& fecal incontinence: nursing management. Saint Louis: Mosby; 2000. p. 29-34.

(2) Abrams P, Blaivas JG, Stanton SL, Andersen JT. The standardization of terminology of lower urinary tract function. Scand J Urol Nephrol 1988; suppl 114:5-26.

(3) Bicalho OJ, Rocha Filho MA, Faria Neto NA. Doenças neurológicas e envelhecimento: disfunções miccionais habitualmente conseqüentes. In: Bruschini H, Kano H, Damião R, editores. I Consenso Brasileiro. Incontinência urinária, uroneurologia, disfunções miccionais. São Paulo: BG Cultural; 1999. p. 55-64. verificadas entre a prevalência e a disúria $(\mathrm{r}=0,19$ e $\mathrm{p}=0,046)$, as infecções urinárias $(\mathrm{r}=0,24 \mathrm{e}$ $\mathrm{p}=0,019)$, o tempo de internação $(\mathrm{r}=-0,32 \mathrm{e}$ $\mathrm{p}=0,002)$ e o sexo masculino $(\mathrm{r}=-0,27$ e $\mathrm{p}=0,008)$. Embora os índices obtidos revelem uma fotografia momentânea da situação, parecem suficientes para apontar a relevância de condição tão subestimada e pouco valorizada em nosso meio, a ponto de não constituir, geralmente, aspecto investigado na avaliação médica ou de enfermagem.

Apesar de casuística tão reduzida, alguns fatos ratificaram os dados de literatura como o predomínio das perdas urinárias entre as mulheres. A idade média similar entre as pessoas de ambos grupos, com e sem IU, contribuiu, mais uma vez, para o rompimento do mito do envelhecimento como fator causal isolado da IU.

As características das perdas urinárias predominantes, por sua vez, não foram indicativas de uma IU grave, o que talvez tenha colaborado para o reduzido número de sentimentos negativos a ela atribuídos bem como a ausência de modificações nas atividades de vida diária e de ocupação. Por outro lado, tem-se a própria hospitalização como fator que poderia estar associado ao aumento de perdas urinárias, o que não foi aqui investigado mas que suscita temática para novas pesquisas.

O mais importante é que se perfila, diante de nós, mais de um terço de uma clientela com perdas urinárias, e cuja realidade certamente repetese em todas as instituições hospitalares, com maiores ou menores índices, gerando mais um problema não resolvido. Abrem-se, assim, novas possibilidades não só para a ampliação dos dados diagnósticos - como este estudo permitiu mas, principalmente, profiláticos através de programas multidisciplinares precoces nos grupos considerados de maior risco.

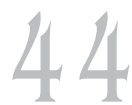

Rev Esc Enferm USP $2005 ; 39(1): 36-45$.
(4) Newman DK. You can run, but you can't hide. In: Newman DK. The urinary incontinence sourcebook. Chicago: Lowell House; 1999. p. 115-9.

(5) URINARY incontinence in adults and chronic management. Guideline Juitline Panel. Clinical Practice Guideline $n^{\circ} 2$. Urinary incontinence in adults. Rockville, MD: US Department of Health and Human Services. Public Health Service. Agency for Health Care Policy and Research, 1996.

(6) Jahn AC. Incontinência urinária: a dimensão do problema na visão de mulheres idosas. [dissertação] Escola de Enfermagem de Ribeirão Preto da USP, 1997. 
(7) O'Donnell PD. The social significance. In: O'Donnell PD. Urinary incontinence in America. Saint Louis: Mosby; 1997. Chap.2, p. 4.

(8) Rocha LC, Rocha FET, Truzzi JCCJ. Tratamento farmacológico da bexiga hiperativa. In: Bruschini $\mathrm{H}$, Kano H, Damião R, editores. I Consenso Brasileiro. Incontinência urinária, uroneurologia, disfunções miccionais. São Paulo: BG Cultural; 1999. p. 45-52.

(9) Steeman E, Defever M. Urinary incontinence among elderly persons who live at home: a literature review. Nurs Clin North Am 1998; 33(3):441-55.

(10) Georgiou A, Potter J, Brocklehurst JC, Lowe D, Pearson M. Measuring the quality of urinary continence care in long-term care facilities: an analysis of outcome indicators. Age and Ageing 2001; 30(1):63-6.

(11) Hoshi T, Nakahara T, Hashimoto S, Hayashi M, Takabayashi K, Miki K et al. Prevalence of urinary incontinence among institutionalized persons aged 60 and over in Japan. [abstract]. Nippon Koshu Eisei Zasshi 1995; 42(7):482-90.

(12) Ando M, Nagamatsu H, Tanizawa A, Terao T, Tsukamoto T, Oshima $\mathrm{H}$ et al. A questionnaire survey on micturation problems among institutionalized elderly. [abstract]. Nippon Hinyokika Gakkai Zasshi 1991; 82(4): 560-4.

(13) Aggazzotti G, Pesce F, Grassi D, Fantuzzi G, Righi E, De Vita D et al. Prevalence of urinary incontinence among institutionalized patients: a cross-sectional epidemiologic study in a midsized city in northern Italy. Urology 2000; 56(2):245-9.

(14) Topinkova E, Sgadari A, Haas T. Urinary incontinence in patients in long-term institutional care: results of an international study in eight countries. [abstract]. Cas Lek Cesk 1997; 136(18): 555-8.

(15) Dias JAA. Epidemiologia da incontinência urinária: prevalência e distribuição na população portuguesa. Instituto de Educação Médica da Faculdade de Ciências Médicas da Universidade Nova de Lisboa, Portugal. [Online]. Disponível em: <http:// www.lem.pt/civ/ecse.html> (02 jun. 2002)

(16) Gavira Iglesias FJ, Caridad Ocerin JM, Molino Martín JP, Valderrama Gama E, López Pérez M, Romero López M et al. Prevalence of psychosocial impact of urinary incontinence in older people of a Spanish rural. J Gerontol A Biol Sci Med Sci 2000; 55:M207-14.

(17) Chaojie L, Andrews GR. Prevalence and incidence of urinary incontinence in the elderly: a longitudinal study in South Australia.[Online] Available from: www.cmj.org/21qk/liuchaojie.htm (12 jun. 2002)

(18) Diokno AC, Brock BM, Bromberg AR. Urinary incontinence in the elderly. Urology 1990; 36(2): 129-38.

(19) Luft J, Vriheas-Nichols AA. Identifying the risk factors for developing incontinence: can we modify individual risk? Geriatr Nurs 1998; 19(2):66-70.
(20) Thompson DL. The national coverage decision for reimbursement for biofeedback and pelvic floor electrical stimulation for treatment of urinary incontinence. J Wound Ostomy Continence Nurs 2002; 29(1):11-9.

(21) DuBeau CE. Urinary incontinence management: new questions for old assumptions. J Am Geriatr Soc 2001; 49(6):829-30

(22) López LO, Sugrañes JC, Escánez PZ, López MO. Incontinência urinária. Rev Rol Enferm 1999; 22(11):4-15.

(23) Blanes L, Pinto RCT, Santos VLCG. Urinary incontinence. Knowledge and attitudes in São Paulo. Braz J Urol 2001; 27(3):281-8.

(24) Stoddart H, Donovan J, Whitley E, Sharp D, Harvey I. Urinary incontinence in older people in the community: a neglected problem? Br J Gen Pract 2001; 51:548-54.

(25) Swithinbank L, Abrams P. Lower urinary tract symptoms in community-dwelling women: defining diurnal and nocturnal frequency and the "incontinence case". BJU Int 2001; 88 (suppl 2):18-22.

(26) Ribeiro RM, Girão MJBC, Santos JFM. Alterações hormonais e perdas urinárias no climatério. In: Bruschini H, Kano H, Damião R, editores. I Consenso Brasileiro. Incontinência urinária, uroneurologia, disfunções miccionais. São Paulo: BG Cultural; 1999. p. 97-101.

(27) Burgio KL, Mathews KA, Engel BT. Prevalence, incidence and correlates of urinary incontinence in healthy middle-aged women. J Urol 1991; 146(5):1255-9.

(28) Badia Llach X, Castro Díaz D, Conejero Sugrañes J. Validez de cuestionário King's Health para la evaluación de la calidad de vida en pacientes com incontinencia urinária. Med Clin (Barc) 2000; 114(17):647-52.

(29) Azevedo GR, Marcondes LHMO, Silva NP, Rios LAS, Kimura M, Santos VLCG. Quality of life of women with stress urinary incontinence. In: Final program and abstracts of 14th Biennial Congress of the World Council of Enterostomal Therapists; 2002 July 7-12 Florence: [s.n.]; 2002. p. 249.

(30) Ferolla EC, Santos VLCG. Self esteem of women with stress urinary incontinence. In: Final program and abstracts of 14th Biennial Congress of the World Council of Enterostomal Therapists; 2002 July 712 Florence: [s.n.]; 2002. p. 250.

(31) Diokno AC, Brock BM, Brown MB, Herzog AR. Prevalence of urinary incontinence and other urological symptoms in the non institutionalized elderly. J Urol 1986; 136(5):1022-5.

(32) Robinson JP. Managing urinary incontinence in the nursing homes: residents' perspectives. J Adv Nurs 2000; 31(1):68-77.

(33) Gallagher M, Pierce LL. Caregivers' and care recipients' perceptions of dealing with urinary incontinence. Rehabil Nurs 2002; 27(1):25-31.
Prevalência da incontinência urinária em adultos e idosos hospitalizados
Correspondência: Vera Lúcia C.G. Santos Depto. Enfermagem Médico-Cirúrgica da EEUSP

Av. Dr. Enéas de Carvalho Aguiar, 419

- Cerqueira César - São Paulo

CEP - 05403-000- SP

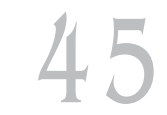

Rev Esc Enferm USP 2005; 39(1):36-45. 